\title{
The Automatic Control System of the Multistage Manual Transmission
}

\author{
Aleksandr Blokhin ${ }^{*}$ \\ Transport systems institute, Nizhny Novgorod State Technical University named after R.E. Alekseev, Nizhny Novgorod, \\ Russian Federation \\ ${ }^{*}$ Corresponding author
}

\begin{abstract}
The paper represents a multi-stage control system of mechanical transmissions of trucks and buses, it considers the process of the system operation when starting the vehicle and the gear shifting. It represents a general diagram of the control program of the multistage manual transmission, built into the microcontroller unit. It describes a functional diagram of the interaction of the main modules with each other and with the engine control unit. It considers the methods used by the system for automatic transmission control.
\end{abstract}

Keywords-multistage transmission; pneumatic actuator; synchronization process; automatic control; command control

\section{TOPICABILITY OF THE AUTOMATIC TRANSMISSION CONTROL}

Improving of the technical level of modern trucks is based on the improvement of operational reliability, competitiveness, increased safety and productivity.

Now these tasks are associated with the degree of saturation of the vehicle with microprocessor control.

The emergence of the global automobile and tractor automation has led to the restructuring of the system design of the components and units of the vehicles. The strength and benefits of such construction of the units is most clearly visible in the example of transmission. Presence and absence of the automatic control (when other components remain the same) leads to a great contrast of the characteristics of the vehicles. Microprocessor control provides much more favorable properties most characteristics of the vehicles: technical, economic and ergonomic ones.

In recent years, there has been an increase in the number of stages in manual transmissions (for example, on road trains up to 16-20). This leads to more frequent gear shifting, which increases physical and psychological stress of the driver. At the same time increase of the speed and amount of road transport requires the driver's attention to the rapidly changing road conditions.

Providing of safety and high performance of vehicles in these conditions can be performed by automated control of mechanical multi-stage transmission, in the first place the transmission and the clutch. Microprocessors, capable of operating in complicated environments of the vehicle performance, allow not only improving of the accuracy of control, but also increasing the number of information parameters, thus implementing more flexible control logic. As a result, the automatic transmission control improves efficiency of the engine power, leads to reduction of fuel consumption, and reduces the driver's fatigue.
Currently, for creation of semi-automatic and automatic transmissions, along with the use of hydro-mechanical and planetary gearboxes, stage synchronized gearboxes are used. They have such positive qualities as a small weight and size, low cost, high reliability, ease of repair and service and high performance are used. Now electro-pneumatic actuators for the shift control have the increasing development in the synchronized gearboxes. It happens due to the presence of compressors required for the control of brakes, doors and etc. on trucks and buses.

When using distance electro-pneumatic actuator, except for facilitating and simplifying of the vehicle control we reduce the vehicle's weight because it eliminates traction, joints, and other units, and expands design possibilities of the vehicle. Furthermore, reducing the time loss for gear shifting we improve the dynamic performance of the vehicle. Due to the widespread use of pneumatic actuators in general engineering their theory and calculation are well developed. In this regard there is a lot of literature.

Comparing microprocessor control systems with their mechanical prototypes by technical properties is easy to see that the microprocessor control systems are much better not only in their general characteristics, but also in terms of quality and design.

Complexes with microprocessor control are not much more expensive than their mechanical prototypes, but on in quality to price ratio they surpass them greatly.

In this regard, the NNSTU named after R. E.Alekseev developed multi-stage control system $(6,8$, and 16-speed) transmissions for trucks and buses.

\section{GENERAL DESCRIPTION OF THE TRANSMISSION CONTROL SYSTEM}

Transmission control system is a hardware-software mechatronic complex, allowing to solve problems of the gear shifting throughout the range of operating modes of the vehicle based on the algorithm (law) embedded in the control unit. Law of the automatic shift control is dependence of the moments of gear shifting (as from lower to higher transmission and vice versa) from the main factors - the vehicle speed and the engine load - the position of the accelerator pedal.

Figure 1 shows the control system of a 16-stage transmission vehicle. The basis of the control unit (position1) is the microcontroller STM32F103VBT6 with the core Cortex-M3, which establishes a law of automatic gear shifting. For the implementation of the law of automatic control of the control unit (pos.1) the following operations 
must be performed:

1. Measurement of the physical quantities necessary for the algorithm selection and gear shift, namely:

- The rotating speed of the output shaft of the engine (position 12), 10)

- The rotating speed of the transmission countershaft (pos.

- The rotating speed of the output shaft of the transmission (pos. 7)

- The absolute position of the accelerator pedal (pos. 18).

2. Determining of the position of the controller lever of the transmission control via scanning of the microswitch sensors (Pos. 5).

3. Determining the position of the transmission mechanism by means of scanning of position sensors of the corresponding mechanism (the clutch control mechanism pos. 14,15 , the main gear control mechanism - pos. 9, the control mechanism of the divider - pos. 8, the control mechanism of the splitter - pos. 11).

4. The determination of the position of the friction disc of the transmission clutch by measuring of the displacement sensor (pos. 19).

5. The issue of the control actions on electro-valves of the gear transmission (main gearbox, splitter, and divider) and control of the friction clutch mechanism.

6. Transfer of the data about the current state of control and modes of transmission to the display (pos. 2).

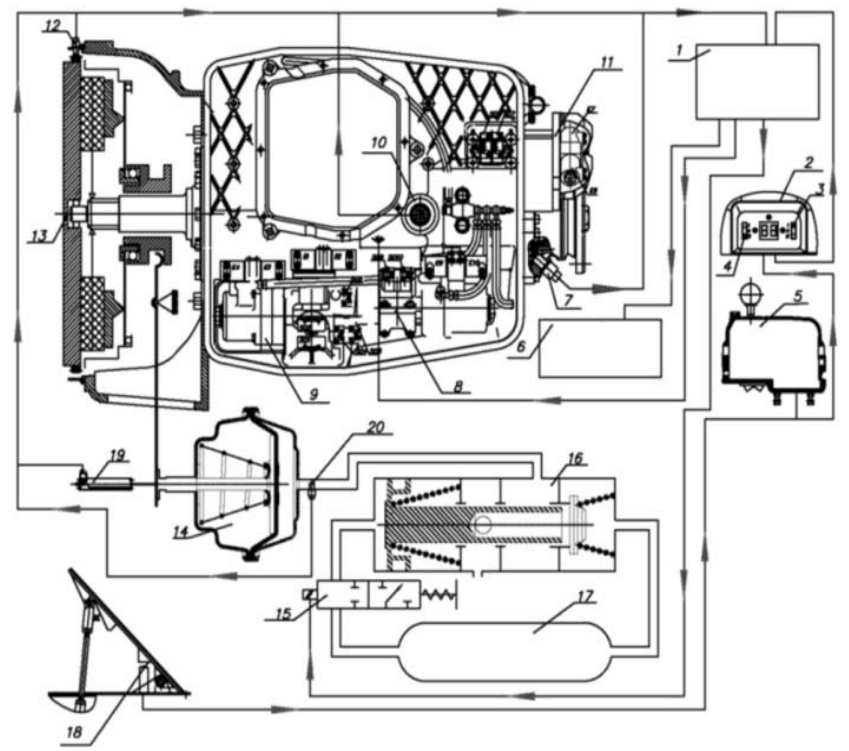

FIGURE I.

THE GENERAL SCHEME OF THE TRANSMISSION AND CLUTCH CONTROL

1 - transmission control unit; 2 - display unit; 3 - automatic control for various conditions; 4 - adjustment to the maximum rate of pressure; 5 controller; 6 - fuel control unit; 7 - output shaft speed sensor; 8 - divider control mechanism; 9 - main gear control mechanism; 10 -intermediate shaft speed sensor; 11 - splitter control mechanism; 12 - engine speed sensor; 13 - clutch; 14 - pneumatic chamber;15 - electro-pneumatic clutch control valve; 16 - accelerating pneumovalve; 17 - receiver;18 - accelerator pedal;19 - clutch plug motion sensor; 20 - pressure sensor in the pneumatic chamber.
Let's consider the process of the vehicle starting and gear shifting (Figure 1). When choosing the gear for starting on the controller (Pos. 5) the control unit (Pos. 1) energizes the solenoid valve (pos. 15) of the acceleration of the clutch mechanism, which allows to supply the air from the receiver into the cavity of the piston acceleration mechanism and open the injection valve through which the air pressure is supplied to the power pneumatic chamber (pos. 14) and the clutch turns on, and then an appropriate gear in the divider control mechanisms (pos. 8), the main gearbox (pos. 9) and the splitter (pos. 11) also turns on. The driver presses the accelerator pedal (pos. 18) the process of the vehicle starting begins. Wherein the control unit modulates the signal to the solenoid valve (pos. 15) while maintaining a certain pressure in the pneumatic chamber, which is proportional to the angular position of the accelerator pedal (pos. 18). In accordance with the pressure in pneumatic chamber the clutch fork takes a definite position (its position is monitored by the displacement sensor pos. 19) and other kinematically related parts, including the clutch pressure plate, thereby defining a gap between the driving and the driven parts of the clutch, and consequently the friction torque in the clutch. This establishes proportionality between the accelerator pedal position (pos. 18) and the point of friction in the clutch, which is necessary for a smooth vehicle starting.

In the process of driving the driver provides the required transmission acting on the controller (Pos. 5, Figure 1). The process of shifting gears when driving is similar to the process of starting, with the difference that starting from the seventh transmission the need for a smooth connection of the driving and driven parts of the clutch is not required.

With automatic control the process of starting and gear shifting is performed in accordance with the law of clutch control, the main gear and the divider. To avoid the overtwist of the engine in the automatic mode shifting to a lower range of the splitter at speeds above $30 \mathrm{~km} / \mathrm{h}$. is prohibited Actuators of the control system in automatic and team modes ar standardized, so the control algorithms in these modes are similar.

\section{The Methods Used By the SYSTEM FOR THE AUTOMATIC TRANSMISSION CONTROL}

The multistage transmission control in the automatic control mode requires software linking the central transmission control unit to the engine control unit and an information display which can be integrated into the control panel. The central microcontroller based on the embedded Law of automatic gearshift in main gearbox and a divider and signals from the sensors of the engine rotation speed, the output shaft rotation speed, the sensor of the angular position of the gas pedal, the sensor of the rod clutch fork position generates control signals to control the friction clutch mechanisms, the main gear, the divider, the splitter, and to the fuel supply cut-off engine.

In the automatic transmission control the following methods are used:

1) The method of adjusting the distance between the discs of the friction clutch depending on the gas pedal position and the engine rotation speed.

The method is continuous monitoring and comparison of the real state of the clutch fork stock and the theoretical value, the programmed functional relationship with the position of 
the gas pedal and the engine rotation speed. Depending on the differences of values of these parameters the control unit generates signals to turn on or turn off the solenoid valves that control the supply and exhaust of the air from the power control chamber mechanism of the friction clutch, including with the use of pulse-width modulation (PWM).

2) The methods of the data exchange via CAN bus according to standard SAE 1939. The CAN protocol is implemented in the microcontroller and is programmed with special function registers.

3) Standard methods of transformation of the signals, of the sent / received data as either byte arrays or as defined structures or classes.

4) Method of power protection against short circuits in the sensor circuits of transmission and the friction clutch control mechanism

This method is implemented by the fact that the power supply of external sensors (microswitches) is made through the limiting resistors. The input circuits of sensors of the transmission mechanisms and of the friction clutch made of high-threshold logic circuit, eliminating interference in the input circuits, active signal for which is the presence of the control unit inputs the voltage of high level, at least $18 \mathrm{~V}$. This eliminates false information in case of disconnection (opening) or short on "weight" of external circuits of the transmission and mechanisms of the clutch control (with open microswitch, in case of the open circuit and short circuit, the low level signal "log. 0" will be shown at the input of the device, and only in case of the closed microswitch the passage of the high-level voltage across the circuit, high level signal "log. 1" will be shown at the input of the device). Signals from the sensors via signal conditioners are applied to inputs of the microcontroller and written on internal registers for further processing by the software.

5) The method of processing signals from inductive rotation speed sensors

The signals from the inductive engine rotation speed sensor, an intermediate shaft and the output shaft of the main gear in a sine wave of variable frequency and amplitude (the amplitude of the sensor signal depends on the speed of passage of gear teeth through the magnetic field sensor) is applied to the voltage comparator, after which the generated signals of rectangular form arrive at the inputs of the programmable counters. When a signal gets on counter input on the leading edge, the main program is interrupted and the subprogram of the interruption processing starts counting of the time interval; then at the next impulse once again an interrupt occurs and the subprogram of the interruption processing captures the counter, forming a period of the signal, and determines rotation speed as the value inverse to the period.

\section{6) Electro-pneumatic valves control method}

When you turn on or off electro pneumatic valve the information is written to the output register of the microcontroller and appropriate logic signals appear. To amplify signals (of the voltage and current) a special chip is used.

\section{THE GENERAL SCHEME OF THE TRANSMISSION PROGRAM CONTROL}

The program multistage transmission control in automatic mode consists of several modules. Communication between them is provided by the main module. Functional diagram of the interaction between the main modules with each other, as well as with the engine control unit is shown in Figure 2 .

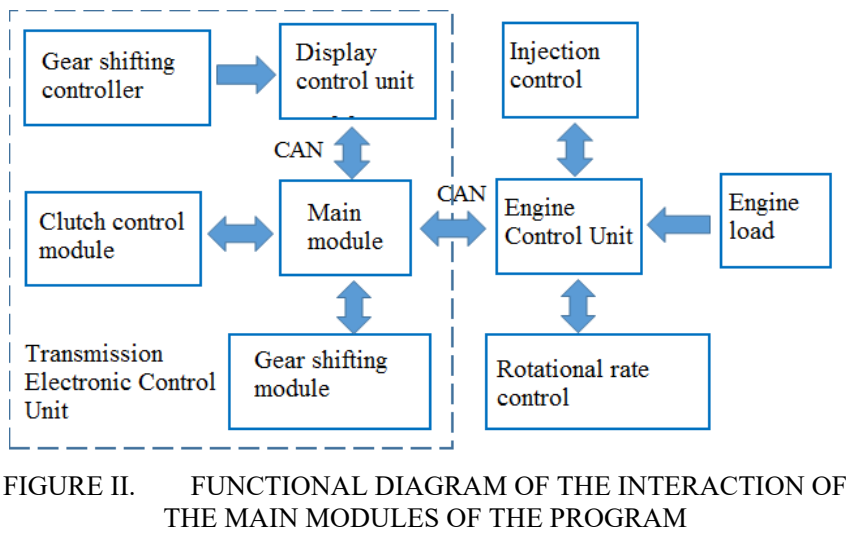

The general scheme of the multistage transmissions control program is made in such a way that when the program starts the main module is used for entering of the main variables that will be used in the modules of the gearswitching control and clutch control, then there is a call of standard libraries designed for preparation of the modules of the gear-switching control and the clutch control, then these modules are initialized and activated. The control module of the gear-switching is performed continuously until the completion of the program, and the clutch control module is performed is interrupted with a period of once per millisecond maximum.

The clutch control module (Figure 3 ) in real-time mode there is monitoring and control of the distance between the clutch plates, that provides smooth and precise on / off switching of the clutch depending on the position of the gas pedal and the engine rotation speed changes when starting the vehicle and shifting gears.

The algorithms for automatic on / off gear switching of the main transmission gearbox, the divider and the splitter are implemented in the control module (Figure 3 ) in accordance with the Law on automatic transmission. Through a constant exchange of information with the main module in the real-time mode based on the analysis of information on engine rotation speed, load, fuel supply and data from the controller (on which the automatic control mode is selected) we make a decision a decision on the possibility and necessity of gear shifting.

The module of work with the display unit (Figure 3) with the help of the LEDs and indication of certain characters allows the operator (driver) to have visual information as input signals to the switching of a particular gear, and directly on switched positions in each of the 3 gears (the main gearbox, the divider and the splitter) and the clutch control mechanism. The module of work with the display unit has its own microcontroller that provides a permanent data exchange with the main module on the CAN bus 
The controller (Pos. 5, Figure 1), a setter of a gear for motion in this mode is set to the position corresponding to automatic shifting.

The main module synchronizes the data transfer between the modules of the program and controls the operation of the main control unit of the multi-stage transmission.

To connect the program with other software programs (the engine control unit and the indicator with their microcontrollers) the protocol SAE 1939 is used on the CAN- bus.

\section{PRACTICAL IMPLEMENTATION OF THE CONTROL SYSTEMS}

Laboratory experimental studies of transmission control systems were carried out in the NNSTU named after R. E. Alekseev (Russia) on a special testing stand for multifunction testing of units of mechanical transmissions of the vehicles with automatic or command control ((Patents of the Russian Federation for utility model №154871, №154102).

Figure 3 shows the testing stand with the connected equipment for testing of multi-stage mechanical transmissions.

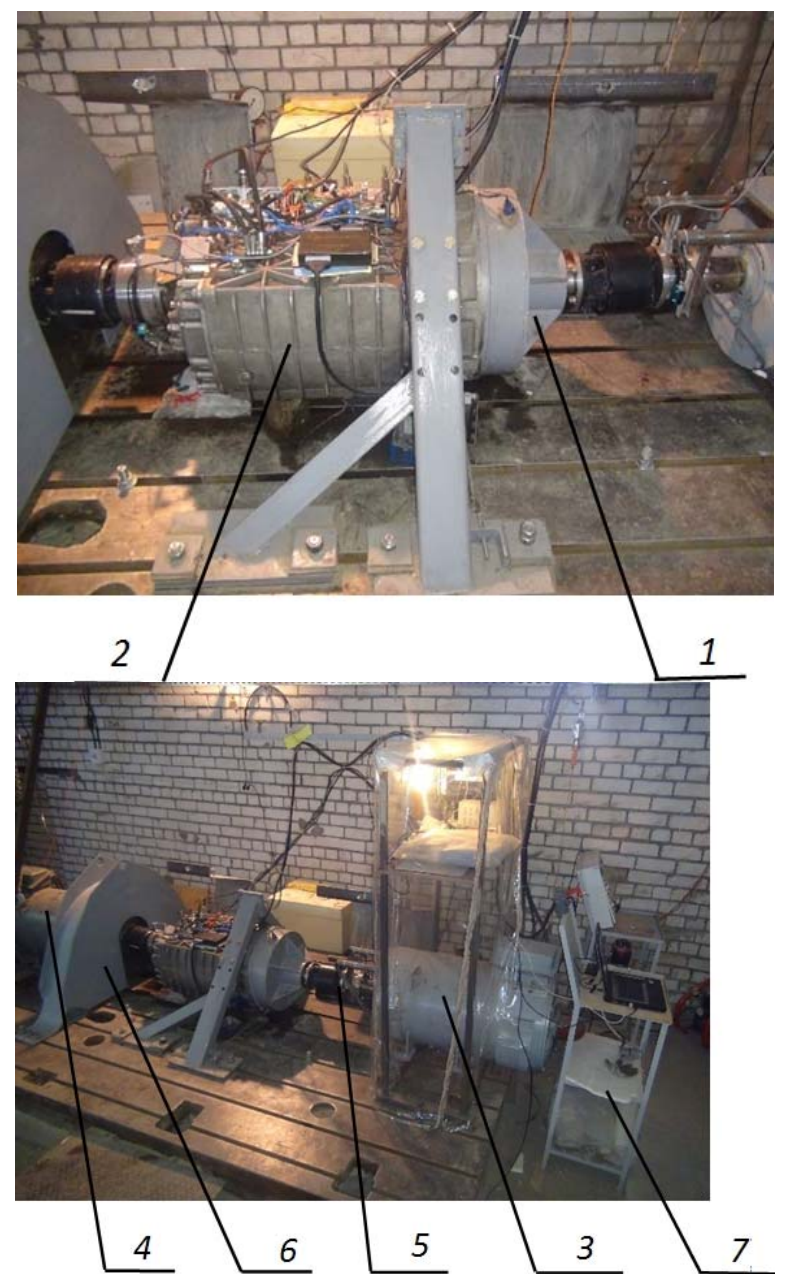

FIGURE III. ESTING STAND CONNECTED TO THE MEASURING EQUIPMENT

1 - clutch control mechanism; 2 - multistage transmission;3 - the driving engine; 4 - generator; 5 - driveline; 6 - inertial mass; 7 - the testing stand remote control
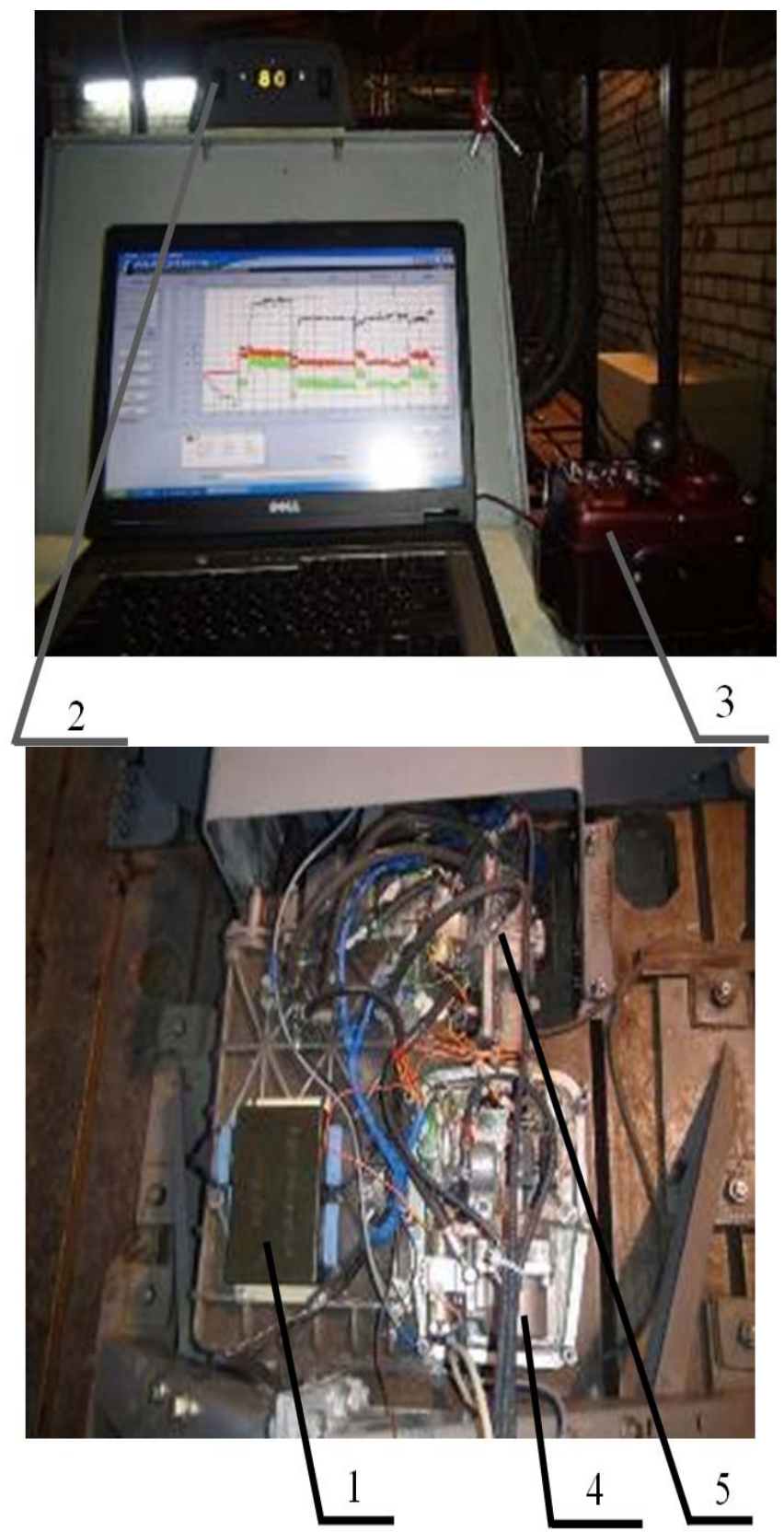

FIGURE IV. LOCATION OF THE MAIN SYSTEMS AND CONTROL MECHANISMS ON THE TESTING STAND

1 - transmission control unit; 2 - display; 3 - controller.

The results of the automatic control systems on the testing stand are described in detail in publications [1-7]. In general, the automatic transmission control system successfully passed laboratory experimental studies on the testing stand and was recommended to be installed on the vehicle.

Experimental samples of the automatic control system of manual transmission were mounted on KAMAZ-65117. Figure 5 shows the arrangement of the display and the controller in the cab of the truck KAMAZ-65117 (Russia). 


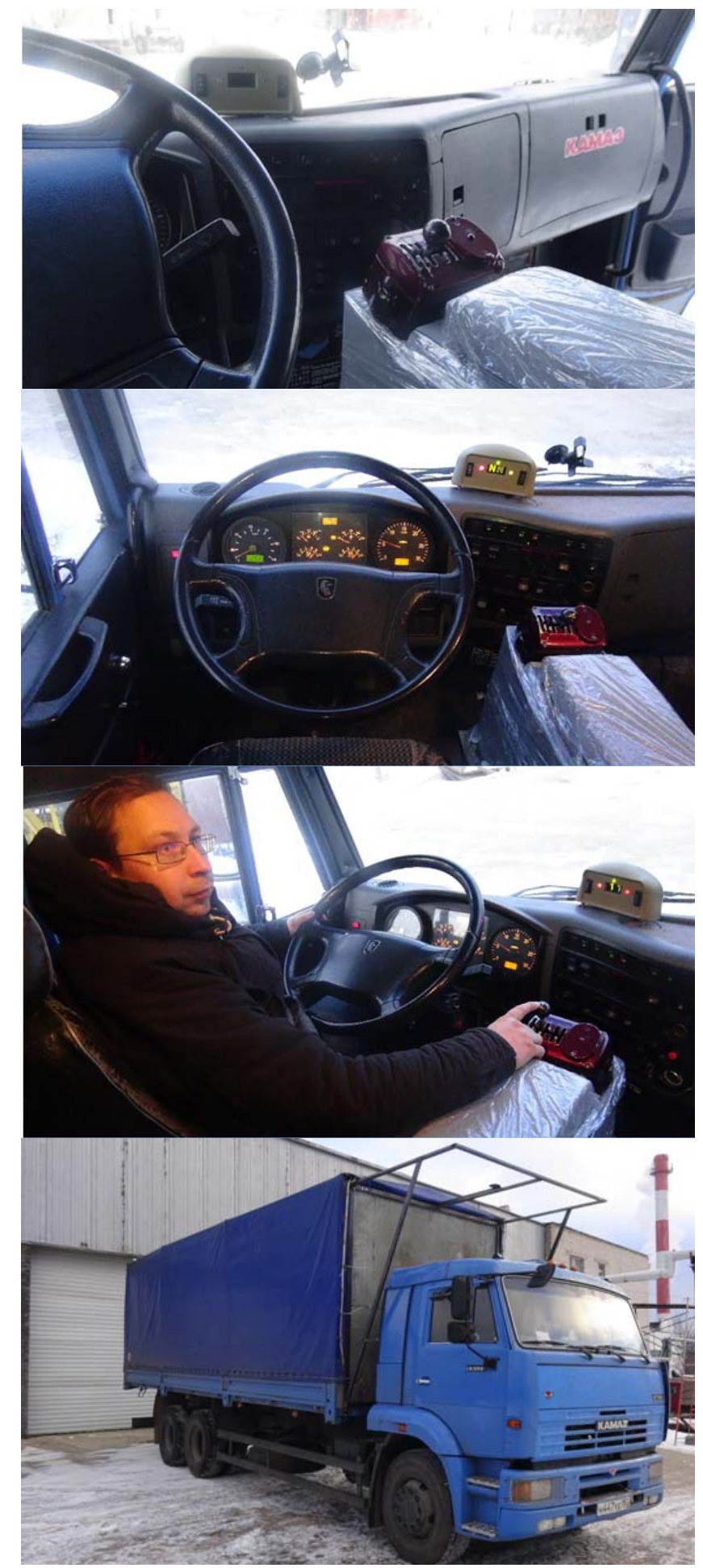

FIGURE V. LOCATION OF THE DISPLAY AND THE CONTROLLER IN THE CAB OF KAMAZ-65117

\section{CONCLUSION}

1. We developed the control system of multistage mechanical transmissions, analyzed the process of the system operation when starting the vehicle and gear shifting.

2. We analyzed the methods used by the system in the process of the automatic transmission control: methods for adjusting the distance between the discs of the friction clutch, depending on the position of the gas pedal and the engine rotation speed, the methods of data exchange on the CANbus, the method of protecting a power supply short-circuit in the sensor circuits of the gearshift and the control mechanism of the friction clutch, method of processing signals from inductive speed sensors and control method of electropneumatic valves.

3. We represented a general diagram of the control program of multistage manual transmission, built into the microcontroller unit. We described a functional diagram of the interaction between the main modules of the program with each other and with the engine control unit.

4. The developed control system of the multistage mechanical transmissions successfully passed laboratory experimental research in the NNSTU named after R.E. Alekseev (Russia) on special testing stand for multifunction testing of the units and aggregates of mechanical transmissions of vehicles with automatic or command control. This control system mounted on a truck KAMAZ65117.

\section{ACKNOWLEDGEMENTS}

It should be noted that the this work was carried out at the NNSTU named after R.E. Alekseev, with financial support from the government in the face of the Russian Ministry of Education under the Federal Program "Research and development on priority directions of the scientifictechnological complex of Russia for 2014-2020, the unique identifier of the project: RFMEFI57414X0040.

\section{REFERENCES}

[1] Blokhin, A. Perspective dimension-types range of mechanical multispeed gearboxes with automatic control system. / A. Blokhin, A. Tumasov, A. Nedyalkov, V. Mankovsky // Proceedings of 2012 International Conference on Advanced Vehicle Technologies and Integration (VTI 2012), July 16-19 2012, Changchun, China, pp.480484, ISBN 978-7-111-39909-4.

[2] A.N. Blokhin, The results of stand tests of sixteen speed transmission with automatic control. / A.N. Blokhin A.P. Nedyalkov, A.E. Kropp // technomag.edu.ru: Education \& Science: electronic science and technology publication. "- $2013 . \quad$ - №12. http://technomag.edu.ru/doc/671097.html.

[3] A.N. Blokhin, The results of computational and experimental studies of efficiency of the multistage mechanical transmission with automatic control / AN Blokhin AP Nedyalkov, AV Tumasov, AM Sox // Actual questions of Mechanical Engineering. Collection of scientific papers. Issue 2 - Minsk: AMI NAS of Belarus, 2013. - S. 171-178, ISSN2306-308.

[4] A. N. Blokhin Process modeling of the transmission with multispeed pneumatic drive clutch control- "Actual problems of Engineering" collection of scientific papers, MI NAS Berarus Minsk, 2014, pp. 139-143.

[5] A. N. Blokhin, A. P. Nedyalkov Development and evaluation of performance electro-pneumatic clutch control mechanism of the vehicle - "Mechanics and Control Processes" volume 2 Materials of the XXXXIV All-Russian symposium on the 90th anniversary of academician V. P. Makeyev, - M.: Russian Academy of Sciences, 2014 pages 51-62.

[6] A. Blokhin, E. Denisenko, A. Kropp, A. Nedyalkov. The Study of the Synchronization Process in the Gearbox with Automatic Control// Applied Mechanics and Materials Vol. 763 (2015) pp 71-77.

[7] A. Blokhin, S. Dobryaev, A. Plekhov, V. Titov. Electromechanical Complex of Test Loading Stand of Multistage Transmissionswith Automatic Control // Applied Mechanics and Materials Vol. 763 (2015) pp 78-85. 